\title{
Improve Peer Cooperation Using Social Networks
}

\author{
Victor Ponce, Jie $\mathrm{Wu}$, and Xiuqi Li \\ Department of Computer Science and Engineering \\ Florida Atlantic University \\ Boca Raton, FL 33431
}

\begin{abstract}
Due to the dynamic nature of P2P systems, it is impossible to keep an accurate history of the transactions that take place while avoiding security attacks such as whitewashing and collusion, and abuse such as freeriding. This is why it is important to develop a mechanism that both rewards cooperative peers and punishes misbehaving peers. Modelling P2P networks as social structures can allow incentive mechanisms to be developed that prevent the negative behaviors mentioned. In a social structure, peers make and receive payments for services provided to and from each other. In this paper we extend a social network algorithm to include the transfer of credit between peers to reduce the path length in queries. We also develop a selection strategy that involves different aspects of peer interactions in a P2P network and a credit transfer mechanism that helps to dis-incent misbehaving peers by taking away credits that they have with good peers and transferring them to more cooperative ones. The simulation results show that our algorithm is effective in reducing the amount of debt between peers, meaning that peers become more cooperative, and shortening the average path length to a satisfied query, while increasing delivery ratio.
\end{abstract}

Keywords: Balance, credit, friendship, incentives, peer-to-peer (P2P), proximity, social network, trust.

\section{INTRODUCTION}

Peer-to-peer (P2P) networks are computer networks consisting of ad hoc connections. These ad hoc connections are formed between individual peers and each peer is both a client and a server. P2P networks are primarily used for the efficient widespread distribution of files, known as file-sharing. The ability to establish ad hoc connections between individuals makes P2P networks popular. The increase of their use has led to more advanced forms of $\mathrm{P} 2 \mathrm{P}$ networks use in a wider range of applications. P2P networks can be classified as structured or unstructured depending on how the data is stored within the network. In this work we focus on unstructured P2P networks. Unstructured P2P networks are less organized and do not have a central server. An advantage of unstructured P2P networks is that joining the network is simple. Also, they are extremely resilient to node join-leave (commonly referred to as turnover), because no special network structure needs to be maintained [7]. Protocols such as BitTorrent [2], Gnutella [5], and eDonkey [8] are just some examples of these filesharing systems. Queries are flooded through the network to find the desired files in unstructured P2P networks. This type of network is also vulnerable to attacks by malicious peers, since the free nature of the network makes it difficult to enfore security.

This work was supported in part by NSF grants ANI 0073736, EIA 0130806, CCR 0329741, CNS 0422762, CNS 0434533, CNS 0531410, and CNS 0626240. Email: vponce@fau.edu, \{jie, xli\}@cse.fau.edu
A peer in a P2P network that consumes many resources but provides few is referred to as a freeloader, leecher, or freerider. It was found that the algorithms used in the original implementation of BitTorrent were not able to effectively reduce the amount of freeriding in the system unless it had few seeds [6]. As shown in [1], more than $70 \%$ of the population in the Gnutella network consume the resources of the network without contributing in return. Another type of malicious behavior is to willfully cheat other peers. Cheating, in this sense, is to disrupt network traffic or knowingly provide corrupt or harmful files. Whitewashing is a term used to describe the action that a peer performs when it leaves the system (discarding its id) and rejoins the network at a later time (with a new id). This behavior is used by peers to "wash" away their previous bad actions.

Social networks are one way to alleviate the problems that are inherent to unstructured P2P networks. Social networks are structures made of nodes that are individuals or organizations. They are used to define relationships (links) between the individuals. The relationships depend on the characteristics of the individuals and their interactions. The characteristics of a peer determine its behavior, which in turn determines the quality of the relationship. P2P networks modelled as social networks include some sort of incentive mechanism so that peers are more inclined to be cooperative. Some measure of contributions, such as credit, is used to quantify the quality of the relationship between peers. Incentive mechanisms reward good peers and punish those that misbehave by giving or taking away credit.

In this paper, we extend an existing incentive mechanism that is successful in isolating freeriders [11]. We include the transfer of credit between peers to reduce the path length in queries. We also develop a selection strategy that involves different aspects of peer interactions in a P2P network. The credit transfer mechanism also helps to dis-incent misbehaving peers by taking away credits that they have with good peers and transferring them to more cooperative ones. We model a P2P network as a social structure where each peer behaves as a person in a society, making judgmental decisions about other members in the society.

The rest of this paper is organized as follows: Section 2 gives some background on some social network models and describes the model we are extending. Section 3 describes our idea in detail. Section 4 describes the simulation environment and the results, and Section 5 concludes the paper. 


\section{RELATED WORK}

There has been much research in $\mathrm{P} 2 \mathrm{P}$ networks using a social structure to improve cooperation by providing good incentives. The idea of modeling a computer network as a society of peers is introduced to solve the principle problems of ad hoc networks, which is that, due to the lack of authority or structure, peers may behave selfishly. This is why criteria are introduced to score peers. All related work are similar in that they use history information and some manner of credit between peers.

In [9], Nandi et al. implemented a transitive trade system where credit is transferred throughout the entire path of a transaction. In their schemes, peers' interactions are described by a relationship where peers have credit and confidence values with those with which they have interacted. A Distributed Hash Table (DHT) is used to find paths to a data source. Their protocol uses the Pastry [10] routing constraint to find nodes with keys that most closely match the requested data key.

In [11], Wang et al. use a social network to model a P2P system. They model a P2P network using a directed graph, where the nodes are peers and the edges are connections between peers. They define a friendship between two peers which is represented by the directed edges in the graph. Each edge has a credit and a payment weight assigned to it, where the credit from one node to another is the payment from the other node to itself, i.e., $C_{i j}=P_{j i}$ where $i$ and $j$ are two nodes in the graph with an edge connecting them. Each node assigns each direct (1-hop) neighbor a credit and payment, and these neighbors are called friends. This information about the data transferred between peers is then used to describe the strength of the friendship. Then, a balance of friendship is used in a decision function to determine routing paths. This has the drawback of not allowing peers to choose a path not only based on direct interaction with their neighbors but also the location of the data they seek. This paper lacks an important decision criterion: location. Disregarding the location of the destination peer implies that all paths have the same cost regardless of their lengths, which also prohibits the selection of the best possible server.

In [3], Feldman et al. develop a model in which users decide whether or not to contribute to a system based on the number of other contributors in the system. If there are too few contributors then the deciding peer will be less willing to participate because of the increased load on itself. The cost of contributing on a peer is the inverse of the total percentage of contributors. This research differs in that different properties of the network are taken into account in routing decisions.

In [13], Zhang et al. define a scheme in which each node is associated with two parameters: money and reputation. Peers exchange money for service and increase their reputations while doing so. There is a central authority that settles disputes between peers when one believes it overpaid or didn't recieve enough service. The central authority is a set of randomly chosen nodes in the network. Similar to other schemes, they classify peers into three different types: honest, selfish, and malicious.

Similar to the aforementioned papers, we have a credit

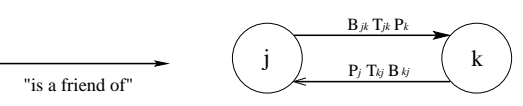

Fig. 1. Representation of balance and trust between peers in a p2p social network. The absolute balance between two nodes is the same in both directions, i.e., $B_{j k}=-B_{k j}$.

system that is used to keep track of the interaction between peers. We introduce two new properties to consider: transferring credit and proximity to data source. Transferring credit will promote proximity routing by allowing those paths that were inaccessible before (because of debt) to be taken.

\section{INCENTIVE MODEL}

The unstructured P2P network is modeled as a directed graph where each vertex, or node, represents a peer in the network. The edges of the graph represent the relationship between two peers, which may also be referred to as a friendship. There are exactly three arcs from one node to another friend node, whose weights are described below. A peer that initiates a search, or query, is referred to as the source and the peer which stores the data that is being queried is called the destination. 1-hop neighbors of a peer are called friends.

There are several criteria that peers use in the selection of friends to query. They are balance, trust, and proximity, which are shown in Figures 1 and 2.

1) balance - $B_{j k}$, the difference between the total amount of service provided by a node $j$ to a node $k$ and the total amount of service provided to node $j$ by node $k$. This amount is cumulative from the first interaction between the two nodes and is used in server selection and decision to serve.

2) trust $-T_{j k}$, the quantitative measurement of the interaction between the nodes $j$ and $k$. The longer the relationship, the higher the trust which means that a long-time friend will have a better chance of serving and, likewise, be served. Trust is used in server selection and decision to serve.

3) proximity - $P_{k}$, the relative distance of a friend node $k$ from the destination node $d$ from current node $i$ 's perspective. Proximity is measured in terms of number of hops from the friend node to the destination and is used only in server selection.

These properties are used by each node in selecting a path to the destination so that two things occur: 1) the network bandwidth is utilized efficiently by routing through paths that are closest to the source, and 2) by using balance and trust between direct neighbors, peers will initially choose the friend that owes it the most, relative to the debt of the other direct neighbors.

We make the following assumptions in our work: First, peers in the network are dynamic, joining and leaving as they please. For ease of programming, when a peer exits the network it is replaced by another peer with the same characteristics. Second, the content of the data being stored and transferred in the network is not the issue in this paper. Therefore, it is possible 


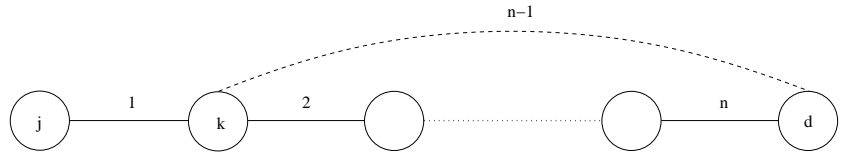

Fig. 2. The proximity of $k$ to $d$, the destination, from $j$ 's perspective is written as $P_{k}$ and is equal to the number of hops from $k$ to $d$, if the total number of hops in one path from $j$ to $d$ through $k$ is $n$ then $P_{k}=n-1$.

for a node to send incorrect data to a requesting node, whether it is due to maliciousness or corrupt data. Security is an issue outside of this work.

\section{A. Interaction Between Peers}

The direct interaction between two peers is captured from two aspects: balance and trust. The balance of the interaction depicts the give-receive relationship between two nodes $j$ and $k$, where the amount $G_{j k}$ given and the amount $R_{j k}$ received can be measured in actual data amount transferred in bytes or packets. The balance also reflects which peer is more contributive. The trust value between two peers shows the amount of interaction between them up to that point in time. In other words, although two peers may have only known each other a relatively short period of time, they may have a high trust value if they have exchanged large amounts of data within that time. As in real-life situations in which people are more willing to trust their helpful friends, peers in a P2P network should also be more willing to trust peers with which they've had more positive interaction. The balance and trust are defined as follows for current node $j$ and friend node $k$ :

$$
\begin{aligned}
B_{j k} & =G_{j k}-R_{j k} \\
T_{j k} & =G_{j k}+R_{j k}
\end{aligned}
$$

where $G_{j k}$ (give) is the total amount of service provided by $j$ to $k$ and $R_{j k}$ (receive) is the total amount of service provided to $j$ by $k$.

\section{B. Path Discovery}

As done traditionally in purely unstructured P2P networks, searching is done by flooding the network with a path discovery message. But, similar to [11], we use an iterative deepening approach in which the combination of depth-first and breadthfirst search is used to minimize network bandwidth consumption. In order to govern the extent of network bandwidth that is consumed in the path discovery phase, a time-to-live (TTL), which is number of hops, is set by the source node and the request is sent to all of its friends. At each hop the TTL is reduced by one. If the friends do not have the data they forward the path discovery message to their friends until the TTL parameter is zero or the data is found. Each time the data is not found, which is detected by a timeout, the source node increases the TTL, if it is less than a specified limit, by one and the query is sent again to all of its friends. Again, the message is forwarded until the TTL is reached or the data is found. All of the peers which are the next hop in a path to the data source are put in a separate list $L$ of friends. Since the path discovery message is of negligble size compared to the amount of data that will be transferred in the credit transfer phase there is no payment given to a friend that forwards the path discovery message.

\section{Server Selection}

The path discovery phase may result in some or many paths being found, or none at all. After possibly multiple paths have been discovered, the source decides which path to take based on the relationship it has with the friends through which paths exist. Source $j$ computes a value $Q_{j k}$ for each friend $k$ in $L$ and chooses the peer with the highest $Q_{j k}$ value to request service:

$$
Q_{j k}=\left(\frac{B_{j k}}{\sum_{i=0}^{f} B_{j i}}\right) w_{1}+\left(\frac{T_{j k}}{\sum_{i=0}^{f} T_{j i}}\right) w_{2}+\left(\frac{\frac{1}{P_{j k}}}{\sum_{i=0}^{f} \frac{1}{P_{j i}}}\right) w_{3}
$$

where $w_{1}+w_{2}+w_{3}=1$ and $f$ is the total number of friends with paths. The weight assigned to each term may vary depending on what is more important in a particular application. If, for example, you want to heavily punish freeriders then $w_{1}$ should be higher. If you want to give more importance to the amount of interaction between friends then $w_{2}$ should be higher. Lastly, if you want to give more importance to relative distance to the data you are looking for then $w_{3}$ should be higher. We experiment with different values for the weights in our simulations to see how system performance differs.

The idea of choosing the next hop based on its nearness to the destination is similar to a mechanism used in [12] where nodes compute heuristic values for their neighbors based on iterative forwarding to choose the least cost path, in terms of energy consumption, to the destination. The cost in P2P networks is the delay in finding your query.

\section{Decision Function}

When a request for service is received by a peer, it decides whether to provide service in the form of supplying the data or forwarding the request according to the output of a decision function. The balance and trust of the friendship are used to calculate the value of the decision function.

The output of the decision function, $F_{k j}$, is a probability deciding whether or not node $k$ should supply service to node $j$, the requesting node. The properties of this decision function are:

- the probability lies within $[0,1]$.

- the probability is a decreasing function of the balance/trust ratio (Figure 3), indicating repayment of services by others because if the ratio is low (balance $\ll$ trust) the probability of supplying service will be high.

The decision function is as follows:

$$
F_{k j}=\frac{1}{2}\left(1-\sin \frac{\pi}{2} \frac{B_{k j}}{T_{k j}}\right)
$$




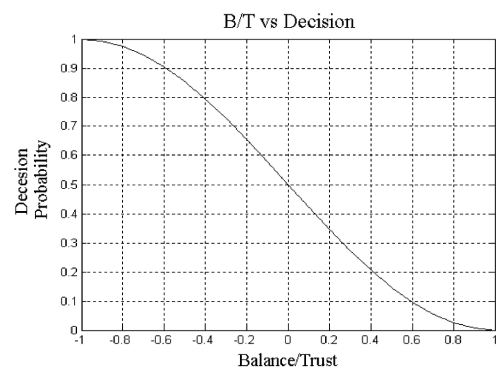

Fig. 3. In the figure, as the balance/trust ratio between two peers $k$ and $j$ increases $\left(\frac{B_{k j}}{T_{k j}} \rightarrow 1\right)$ the probability of peer $k$ serving peer $j$ decreases $\left(D_{k j} \rightarrow 0\right)$, and vice-versa.

This decision function is proven to be effective in reducing collusion in [11]. We propose a transfer of credit mechanism to achieve an improved network balance and delivery ratio. Balancing the network means that we want the friendship balance of all peers to be close to the mean friendship balance of the network such that there are few peers who owe significantly more to others. Simulation results show that the network is more balanced using the credit transfer mechanism.

\section{E. Credit Transfer}

In this work, we propose to allow direct transfer of credit from one peer to another. This transfer of credit aids in balancing relationships between peers. It also helps to make transactions possible between two peers that have direct information about each other but one is in debt of the other and helps peers choose the best path with the criteria mentioned earlier. Credit can only be transferred from a 1-hop peer to another 1-hop peer. The three peers involved in the credit transfer are friends, so each knows about the other. Here is a scenario were credit transfer will allow a peer to serve another where if there had been no credit transfer the transaction would not have occured.

The three peers in the credit transfer are already friends. A simple example of how the credit transfer works is shown in Figure 4. The figure shows that peer $i$ owes peer $j$ an amount 10 , so $B_{i j}=-10$. Peer $k$ owes peer $i$ an amount 10 , so $B_{i k}=$ $-B_{k i}=10$, and $l$ owes $i$ an amount 5 , so $B_{i l}=-B_{l i}=5$. Peer $k$ and peer $j$ are even, so $B_{j k}=B_{k j}=0$. Through the path discovery stage peer $i$ finds that peer $j$ can provide it with the best path. As it is, the request values of $j$ and $l$ are $R_{i j}=0.47$ and $R_{i l}=0.53$. With these values $i$ would normally choose $l$ to query, even though the path through $j$ is closer. So peer $i$ can use the amount owed to it by $k$ to utilize peer $j$ 's services. Peer $i$ zeros the balance that $k$ owes it by adding the difference between $G_{i k}$ and $R_{i k}$ to $R_{i k}$ (to zero the balance) and also adding that amount to $G_{i j}$ (to increase the balance), so that now $R_{i j}=0.79$ which will result in choosing peer $j$. All three peers benefit from the transfer: $i$ is able to use the best path, $j$ is paid what it is owed and an additional amount if $B_{k i}$ was higher than $B_{i j}$, and $k$ does not owe $i$ anymore and it has also gained some trust with $i$. This method of transferring credit retains the history information between the peers so that in future routing decisions the zero
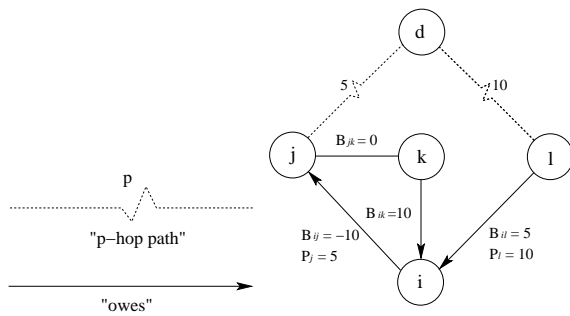

Fig. 4. In the figure, the dotted lines represent the paths from nodes $j$ and $l$ to the destination $d$ and the weight is the hop count, so the proximity value of $j$ and $p$ from $i$ 's perspective are $P_{j}$ and $P_{l}$, respectively. In this example, $G_{i j}=0$ and $R_{i j}=10$, so $T_{i j}=10$ and $G_{i l}=10$ and $R_{i l}=5$, so $T_{i l}=15$ to illustrate the effect that the credit transfer has on the routing decision. If credit transfer is not used, $R_{i j}=0.47$. But, if credit transfer is used $R_{i j}=0.79$, so $j$ will be chosen by $i$ to provide service.

balance does not have a great impact. Using this method, all three peers have to acknowledge the credit transfer.

The credit transfer mechanism is used by the requesting peer when the server denies it service based on the decision function. The requesting peer looks through its list of friends and finds ones that are in debt to it. It then selects the friends that both it and the serving peer have in common. From this peer, the debt is removed and transferred to the serving peer. Then the serving peer acknowledges this transfer and increases the requestors credit by the same amount. In this way, the requestor increases the balance from it to the server and is provided service.

\section{F. Establishing and Breaking Connections}

1) New Connections: After a successful transaction, the peer that initiated the query (requestor) may ask the peer that provided the data (server) to become its friend. There are two factors that are considered in this case: the number of current friends of the server and the distance between the two peers. The following function, which is similar to the one in [11], describes the decision $C_{n e w}$ to form a new connection:

$$
C_{\text {new }}=\min \left[1-\frac{N_{\text {cur }}}{N_{\max }}+\frac{r \times\left(H_{\text {cur }}-1\right)}{H_{\max }}, 1\right]
$$

where $N_{\text {cur }}$ is the current number of friends, $N_{\max }$ is the total number of friends allowed, $H_{c u r}$ is the number of hops that were taken to get to that peer, $H_{\max }$ is the maximum number of hops allowed in a search, and $r$ is a random number, $r \in[0,1]$. This probability function is used after a successful transcation, meaning that the data was transferred from the server back to the requestor, hop by hop. If the distance, in hops, is high the probability to make this new friendship is higher, and vice versa. If the server has few friends then the probability is also higher, and vice versa. The random value $r$ is included because sometimes the server has few empty slots available in its friend list, so the probability of choosing the requestor is mostly influenced by the distance between them.

There is also a mechanism for creating a new connection between two peers that are strangers but have friends in common. In our mechanism, the peer that is asked for friendship asks its friends if they know the stranger peer and weights their responses based on the their relationship. The peer being 
asked also weights its decision using its current number of friends to maximum number of friends ratio. The function to determine whether or not $j$ will accept the new peer $i$ as a friend is denoted as $S_{j i}$ :

$$
S_{j i}=1-\frac{N_{c u r}}{N_{\max }} \times \frac{1}{2} \sum_{k=0}^{f}\left(\frac{B_{j k}}{T_{j k}} \times \frac{B_{k i}}{T_{k i}}\right)
$$

where $f$ is the total number of friends of $j$. This function is used when a new peer enters the network and has no friends. It randomly chooses a peer in its vicinity to request friendship. Peer $j$, the one being asked for friendship, calculates its $S_{j i}$ value about the requesting stranger peer $i$. Peer $j$ takes into consideration its current number of friends as well as their opinions (if any) about $i$.

2) Old Connections: There are times when there is little or no interaction between two peers that are friends. Since peers have a limit on the maximum number of friends they can have at any time, there must be a way to break these ties with the current friends. A simple solution to this is to decay the give and receive amounts by a predetermined value $\lambda$. The connection between two peers will be severed if the balance, $B_{j k}$, between nodes $j$ and $k$ exceeds a threshold. When the balance is higher than the threshold, it means that the amount given, $G_{j k}$, is much greater than the amount received, $R_{j k}$. The give and receive amounts are updated as follows periodically from peer $j$ :

$$
\begin{aligned}
& R_{j k}=R_{j k}-\lambda \\
& G_{k j}=G_{k j}-\lambda
\end{aligned}
$$

for every friend $k$ in $j$ 's friend list that does not serve. Each friend $k$ also updates its information about peer $j$.

The balance and trust amounts can be directly related to the amount of data that is exchanged. So the give and receive amounts are incremented by $\tau$. The give and receive amounts are updated as follows:

$$
\begin{aligned}
& G_{j k}=G_{j k}+\tau \\
& R_{k j}=R_{k j}+\tau
\end{aligned}
$$

for every friend $k$ in $j$ s friend list that served. $\tau$ represents the amount of data or service provided by each friend. Again, both friends update their information about each other so as to keep an accurate record of their transactions.

\section{Simulation Setup and Experimental Results}

In this section we present the simulation settings and demonstrate that our algorithm is successful in accomplishing our goals of reducing path length and debt between peers. First we describe the simulation framework. Then we show the initial settings for our scheme. The settings are listed in Table I.

\begin{tabular}{|c|c||c|c|}
\hline Parameter & Value & Parameter & Value \\
\hline Population Size & 100 & Run time (rounds) & 1000 \\
\hline Max No. Friends & 10 & Ratio Cooperative Peers & $1 / 3$ \\
\hline Ratio Defective Peers & $1 / 3$ & Ratio Decisive Peers & $1 / 3$ \\
\hline Learning Probability & 0.05 & Turnover Rate & 0.01 \\
\hline Initial $G_{j k}$ & 30 & Initial $R_{j k}$ & 30 \\
\hline$\lambda$ (payment decrease) & 2 & $\tau$ (payment increase) & 40 \\
\hline Max Hops & 5 & Cut-off Threshold & 10 \\
\hline
\end{tabular}

TABLE I

TABLE OF PARAMETERS

\section{A. Simulation Framework}

As in [4], our simulation consists of rounds. Each round is a logical time unit in which every peer plays two roles. There are two games: client and server. Every peer decides what role they want to play in the beginning of each round. After the choice is made, some will play as clients and some will play as servers. The peers which are clients may choose whether or not to query, and the peers which are servers may choose whether or not to serve. The credit scores are updated accordingly for serving and non-serving servers each time a query is made. The decision by a server to choose is based on its strategy. There are three types of strategies:

- Cooperative - A peer which always chooses to serve.

- Defective - A peer which always chooses not to serve.

- Decisive - A peer which uses the decision function described in this paper.

At the end of each round each peer will choose to make one of the following actions:

1) Learn - Each peer rates his own strategy. Every time a peer makes a query it updates its strategy's score. When a query is successful it increments the score and when the query fails it decrements the score. At the end of each round, if it chooses to learn, every peer ranks its strategy among its friends and chooses a new strategy with a probability proportional to the difference between its strategy's score and the average of its friends. This probability will be low if the peers strategy is ranked relatively high, so it will more likely keep its current strategy.

2) Exit - If a peer chooses to exit the network, a new peer with the same id and strategy will enter the network to replace it. The rest of the parameters of the peer, such as friends, strangers, and all other values are re-initialized.

3) Remain - A peer may choose to remain in the network and continue with its current strategy. Nothing happens in this case, all parameters for this peer remain the same.

4) Mutate - A peer may randomly choose to mutate to another strategy. All of the peers parameters remain the same with the exception of its strategy.

\section{B. Settings}

Table I shows the default simulation settings. The network consists of 100 peers. The simulation consists of 1000 rounds, where each round is a logical time unit. The maximun number of friends that a peer can have is 10 . Initially, the population in the network is equally divided into three categories of peers: cooperative, defective, decisive. These categories define the 


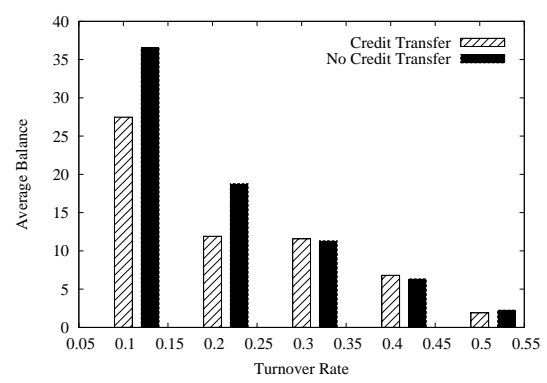

(a) average balance

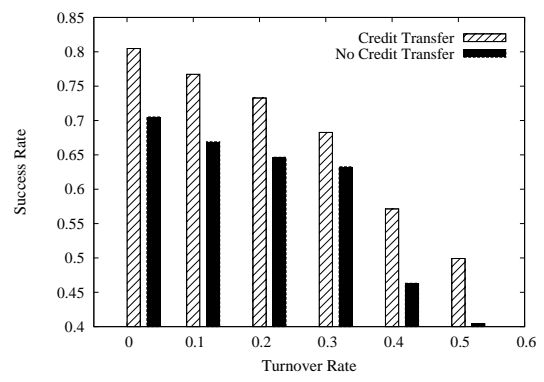

(b) query success ratio

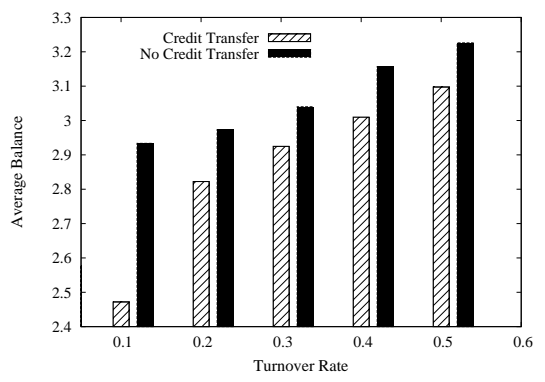

(c) average hop count to data source

Fig. 5. Simulation results

strategy of the peer, which changes throughout the simulation, as described in [11]. The probability to learn and change strategy is set to 0.05 . The turnover rate, or rate at which peers exit the system and are replaced by new peers, is initially set to 0.01 . The Give and Receive amounts are initially set to 30 . These are always the initial values for new friendships. The payment for serving another peers $(\lambda)$ is 2 . The decay amount $(\tau)$ is set to 10 . The maximum number of hops that a query can travel is set to 5. The cut-off threshold for friends who have accumulated too much debt is 10 .

\section{Results}

The weights for the balance, trust, and proximity were varied to find the optimal values. Several different values gave good results, but the weights which gave the best results are: $w_{1}=\frac{1}{2}, w_{2}=\frac{1}{3}$, and $w_{3}=\frac{1}{6}$. The following results were obtained using these values.

Figure 5(a) shows the average balance in the network for different turnover rates with all other values set to default. It shows that our algorithm does have an an average balance closer to 0 . This means that throughout the experiment the average debt of the peers in the network is lower. As the turnover rate increases the average balance for our algorithm is about the same as the algorithm without credit transfer. The reason for this is that when more peers leave and new ones come into the network the Give and Receive amounts are initialized and the balance is 0 , which leaves little room for balancing the debt between friends using credit transfer.

Figure 5(b) shows the average query satisfaction rate for the two different algorithms. As the figure shows, the satisfaction rate using our algorithm is higher at any turnover rate. Even at a 50\% turnover rate, which is realistic in a real P2P system, our algorithm with credit transfer and friend proximity consideration has a query satisfaction rate of close to $50 \%$.

Figure 5(c) shows the average number of hops to the data source node in the query. Clearly, the number of hops to the data source is reduced by using credit transfer and the hop count metric. Our algorithm works exceptionally well at low turnover rates because it uses history information.

\section{CONCLusion ANd Future Work}

Our algorithm accomplished two goals: 1) The overall balance of the network was reduced so that there were fewer peers who had higher debts than the average of the entire network, and 2) The average number of hops to the desired file was reduced. These factors are important because having a lower debt in the network means that there is cooperation.

The first goal can help prevent freeriders from taking advantage of those peers who cooperate. Peers that do not cooperate are eventually isolated from those that do cooperate. The second goal proves that, by allowing the selection of servers to be two-sided, the data can be found faster. The use of path length information as well as history about peer relationships increases the success ratio of queries and reduces the query path length.

In the future we plan to extend our algorithm to include secure and verifiable credit transfer between peers that are not friends. It is difficult to accomplish such a thing because one of those peers may deny ever having transferred credit, without the use of authentication devices such as certificates. There are several factors to be taken into consideration since in P2P networks there is a high turnover rate. History about credit transfers and the amount of storage allocated to it is one factor. Risk of never regaining transferred credit is another consideration.

\section{REFERENCES}

[1] E. Adar and B. A. Huberman. Freeriding on gnutella. First Monday, 5(10), October 2000.

[2] B. Cohen. http://www.bittorrent.org/bittorrentecon.pdf.

[3] M. Feldman, M. Ahamad, C. Papadimitriou, J. Chuang, and I. Stoica. Free-riding and whitewashing in peer-to-peer systems. In Proceedings of the SIGCOMM'04 Workshop, August 2004.

[4] M. Feldman, K. Lai, I. Stoica, and J. Chuang. Robust incentive techniques for peer-to-peer networks. In Proceedings of the 5th ACM Conference on Electronic Commerce, May 2004.

[5] Gnutella. http://www.gnutella.com.

[6] S. Jun and M. Ahamad. Incentives in bittorrent induce freeriding. In Proceedings of ACM SIGCOMM, August 2005.

[7] X. Li and J. Wu. Searching techniques in peer-to-peer networks. In Handbook of Theoretical and Algorithmic Aspects of Ad Hoc, Sensor, and Peer-to-Peer Networks, pages 613-642, 2006.

[8] Merkur. http://www.emule-project.net/home/perl/general.cgi?l=1.

[9] A. Nandi, T. Ngan, P. Druschel, and D. Wallach. Scrivener: Providing incentives in cooperative content distribution systems. November 2005.

[10] A. Rowstron and P. Druschel. Pastry: Scalable, decentralized object location and routing for large-scale peer-to-peer systems. In Proceedings of the 18th IFIP/ACM Conference on Distributed Systems Platforms (Middleware 2001), November 2001.

[11] W. Wang, R. Yuan, and L. Zhao. Improving cooperation in peer-to-peer systems using social networks. In Proceedings of IEEE IPDPS 2006, 2006.

[12] Y. Yu, R. Govindan, and D. Estrin. Geographical and energy aware routing: a recursive data dissemination protocol for wireless sensor networks. Technical report, UCLA Computer Science Department UCLA/CSD-TR-01-0023, May 2001.

[13] Z. Zhang, S. Chen, and M. Yoon. March: A distributed incentive scheme for peer-to-peer networks. In Proceedings of IEEE INFOCOM'07, May 2007. 